\title{
Erratum to: Mindfulness and Self-Compassion: Exploring Pathways to Adolescent Emotional Well-Being
}

\author{
Karen Bluth · Priscilla W. Blanton
}

Published online: 20 October 2013

(C) Springer Science+Business Media New York 2013

Erratum to: J Child Fam Stud

DOI 10.1007/s10826-013-9830-2

The original version of this article unfortunately contained a mistake. The presentation of Tables 4 and 5 was incorrect. The corrected Tables 4 and 5 are given below.

The online version of the original article can be found under doi:10.1007/s10826-013-9830-2.

\footnotetext{
K. Bluth $(\square)$

Program on Integrative Medicine, Department of Physical

Medicine and Rehabilitation, The University of North Carolina,

CB \#7200, Chapel Hill, NC 27599, USA

e-mail: bluth@med.unc.edu

P. W. Blanton

Department of Child and Family Studies, University of

Tennessee-Knoxville, 1215 W. Cumberland Ave., Knoxville,

TN, USA

e-mail: pblanton@utk.edu
} 
Table 4 A priori model with self-compassion as mediator

\begin{tabular}{|c|c|c|c|c|}
\hline \multirow[t]{2}{*}{ DV } & \multirow{2}{*}{$\frac{\text { Direct model }}{\text { CAMM } \rightarrow \text { DV }}$} & \multicolumn{3}{|l|}{ Mediated model } \\
\hline & & $\mathrm{CAMM} \rightarrow \mathrm{DV}$ & $\mathrm{CAMM} \rightarrow \mathrm{SCS}$ & $\mathrm{SCS} \rightarrow \mathrm{DV}$ \\
\hline PA & $.26^{*}$ & .20 (ns) & $.59 * *$ & $.10(\mathrm{~ns})$ \\
\hline NA & $-.61 * *$ & $-.36 * *$ & $.59 * *$ & $-.42 *$ \\
\hline SLSS & $.67 * *$ & $.56 * * *$ & $.59 * *$ & $.20(\mathrm{~ns})$ \\
\hline PSS & $-.61 * * *$ & $-.31 * * *$ & $.59 * *$ & $-.52 * * *$ \\
\hline
\end{tabular}

CAMM Children and Adolescent Mindfulness Measure, SCS Self-compassion Scale, PA positive affect, NA negative affect, SLSS Student Life Satisfaction Scale, PSS Perceived Stress Scale

* Correlation is significant at the .05 level (2-tailed), ** correlation is significant at the .01 level (2-tailed), *** correlation is at the .001 level (2tailed)

Table 5 Alternate model with mindfulness as mediator

\begin{tabular}{|c|c|c|c|c|}
\hline \multirow[t]{2}{*}{ DV } & \multirow{2}{*}{$\frac{\text { Direct model }}{\mathrm{SCS} \rightarrow \mathrm{DV}}$} & \multicolumn{3}{|c|}{ Mediated model } \\
\hline & & $\mathrm{SCS} \rightarrow \mathrm{DV}$ & $\mathrm{SCS} \rightarrow \mathrm{CAMM}$ & $\mathrm{CAMM} \rightarrow \mathrm{DV}$ \\
\hline PA & $.22(\mathrm{~ns})$ & $.10(\mathrm{~ns})$ & $.59 * * *$ & $.20(\mathrm{~ns})$ \\
\hline NA & $-.64 * * *$ & $-.42 * * *$ & $.59 * * *$ & $-.36^{* *}$ \\
\hline SLSS & $.52 * * *$ & $.19(\mathrm{~ns})$ & $.59 * * *$ & $.56^{* * *}$ \\
\hline PSS & $-.70 * * *$ & $-.52 * * *$ & $.59 * * *$ & $-.31 * *$ \\
\hline
\end{tabular}

CAMM Children and Adolescent Mindfulness Measure, SCS Self-compassion Scale, PA positive affect, NA negative affect, SLSS Student Life Satisfaction Scale, PSS Perceived Stress Scale

* Correlation is significant at the .05 level (2-tailed), ** correlation is significant at the .01 level (2-tailed), *** correlation is at the .001 level (2tailed) 